\title{
Nonoccurrence of the Lavrentiev phenomenon for nonconvex variational problems
}

\author{
Alexander J. Zaslavski \\ Department of Mathematics, The Technion-Israel Institute of Technology, 32000 Haifa, Israel
}

Received 25 March 2004; received in revised form 21 June 2004; accepted 6 October 2004

Available online 7 April 2005

\begin{abstract}
In this paper we establish nonoccurrence of the Lavrentiev phenomenon for two classes of nonconvex variational problems. For the first class of integrands we show the existence of a minimizing sequence of Lipschitzian functions while for the second class we establish that an infimum on the full admissible class is equal to the infimum on a set of Lipschitzian functions with the same Lipschitzian constant.
\end{abstract}

(C) 2005 L'Association Publications de l'Institut Henri Poincaré. Published by Elsevier B.V. All rights reserved

\section{Résumé}

Dans cet article nous démontrons que le phénomène de Lavrentiev pour deux classes de problèmes variationnels non convexes ne peut avoir lieu. Nous montrons, pour la première classe d'intégrands, l'existence d'une suite minimisante de fonctions lipschtziennes, alors que pour la seconde classe, nous démontrons qu'un infimum sur toute la classe admissible est égale a l'infimum sur un ensemble de fonctions lipschtziennes, avec la même constante de Lipschtiz.

(C) 2005 L'Association Publications de l'Institut Henri Poincaré. Published by Elsevier B.V. All rights reserved

MSC: 49J27

Keywords: Banach space; Integrand; Lavrentiev phenomenon; Variational problem

\section{Introduction}

The Lavrentiev phenomenon in the calculus of variations was discovered in 1926 by M. Lavrentiev in [9]. There it was shown that it is possible for the variational integral of a two-point Lagrange problem, which is sequentially weakly lower semicontinuous on the admissible class of absolutely continuous functions, to possess an infimum on the dense subclass of $C^{1}$ admissible functions that is strictly greater than its minimum value on the admissible

E-mail address: ajzas1@tx.technion.ac.il (A.J. Zaslavski).

0294-1449/\$ - see front matter (C) 2005 L'Association Publications de l'Institut Henri Poincaré. Published by Elsevier B.V. All rights reserved doi:10.1016/j.anihpc.2004.10.004 
class. Since this seminal work the Lavrentiev phenomenon is of great interest. See, for instance, $[1-4,6-8,10-12]$ and the references mentioned there. Mania [11] simplified the original example of Lavrentiev. Ball and Mizel $[3,4]$ demonstrated that the Lavrentiev phenomenon can occur with fully regular integrands. Nonoccurrence of the Lavrentiev phenomenon was studied in [1,2,7,8,10,12]. Clarke and Vinter [7] showed that the Lavrentiev phenomenon cannot occur when a variational integrand $f(t, x, u)$ is independent of $t$. Sychev and Mizel [12] considered a class of integrands $f(t, x, u)$ which are convex with respect to the last variable. For this class of integrands they established that the Lavrentiev phenomenon does not occur. In this paper we establish nonoccurrence of Lavrentiev phenomenon for two classes of nonconvex nonautonomous variational problems with integrands $f(t, x, u)$. For the first class of integrands we show the existence of a minimizing sequence of Lipschitzian functions while for the second class we establish that an infimum on the full admissible class is equal to the infimum on a set of Lipschitzian functions with the same Lipschitzian constant.

Assume that $(X,\|\cdot\|)$ is a Banach space. Let $-\infty<\tau_{1}<\tau_{2}<\infty$. Denote by $W^{1,1}\left(\tau_{1}, \tau_{2} ; X\right)$ the set of all functions $x:\left[\tau_{1}, \tau_{2}\right] \rightarrow X$ for which there exists a Bochner integrable function $u:\left[\tau_{1}, \tau_{2}\right] \rightarrow X$ such that

$$
x(t)=x\left(\tau_{1}\right)+\int_{\tau_{1}}^{t} u(s) \mathrm{d} s, \quad t \in\left(\tau_{1}, \tau_{2}\right]
$$

(see, e.g., [5]). It is known that if $x \in W^{1,1}\left(\tau_{1}, \tau_{2} ; X\right)$, then this equation defines a unique Bochner integrable function $u$ which is called the derivative of $x$ and is denoted by $x^{\prime}$.

We denote by mes $(\Omega)$ the Lebesgue measure of a Lebesgue measurable set $\Omega \subset \mathbb{R}^{1}$.

Let $a, b \in \mathbb{R}^{1}$ satisfy $a<b$. Suppose that $f:[a, b] \times X \times X \rightarrow \mathbb{R}^{1}$ is a continuous function such that the following assumptions hold:

$$
f(t, x, u) \geqslant \phi(\|u\|) \quad \text { for all }(t, x, u) \in[a, b] \times X \times X,
$$

where $\phi:[0, \infty) \rightarrow[0, \infty)$ is an increasing function such that

$$
\lim _{t \rightarrow \infty} \phi(t) / t=\infty
$$

(A2) for each $M, \epsilon>0$ there exist $\Gamma, \delta>0$ such that

$$
\left|f\left(t, x_{1}, u\right)-f\left(t, x_{2}, u\right)\right| \leqslant \epsilon \max \left\{f\left(t, x_{1}, u\right), f\left(t, x_{2}, u\right)\right\}
$$

for each $t \in[a, b]$, each $u \in X$ satisfying $\|u\| \geqslant \Gamma$ and each $x_{1}, x_{2} \in X$ satisfying

$$
\left\|x_{1}-x_{2}\right\| \leqslant \delta, \quad\left\|x_{1}\right\|,\left\|x_{2}\right\| \leqslant M
$$

(A3) for each $M, \epsilon>0$ there exists $\delta>0$ such that

$$
\left|f\left(t, x_{1}, y_{1}\right)-f\left(t, x_{2}, y_{2}\right)\right| \leqslant \epsilon
$$

for each $t \in[a, b]$ and each $x_{1}, x_{2}, y_{1}, y_{2} \in X$ satisfying

$$
\left\|x_{i}\right\|,\left\|y_{i}\right\| \leqslant M, \quad i=1,2
$$

and

$$
\left\|x_{1}-x_{2}\right\|,\left\|y_{1}-y_{2}\right\| \leqslant \delta .
$$

Remark 1.1. If $X=\mathbb{R}^{n}$, then (A3) follows from the continuity of $f$.

Let $z_{1}, z_{2} \in X$. Denote by $\mathcal{B}$ the set of all functions $v \in W^{1,1}(a, b ; X)$ such that $v(a)=z_{1}, v(b)=z_{2}$. Denote by $\mathcal{B}_{L}$ the set of all $v \in \mathcal{B}$ for which there is $M_{v}>0$ such that

$$
\left\|v^{\prime}(t)\right\| \leqslant M_{v} \quad \text { for almost every } t \in[a, b] .
$$


Clearly for each $v \in \mathcal{B}$ the function $f\left(t, v(t), v^{\prime}(t)\right), t \in[a, b]$, is measurable. We consider the variational problem

$$
I(v):=\int_{a}^{b} f\left(t, v(t), v^{\prime}(t)\right) \mathrm{d} t \rightarrow \min , \quad v \in \mathcal{B},
$$

and establish the following result.

\section{Theorem 1.1.}

$$
\inf \{I(v): v \in \mathcal{B}\}=\inf \left\{I(v): v \in \mathcal{B}_{L}\right\} .
$$

Theorem 1.1 is proved in Section 3.

It is not difficult to see that the following propositions hold.

Propsotion 1.1. Let $\phi:[0, \infty) \rightarrow[0, \infty)$ be an increasing function such that $\lim _{t \rightarrow \infty} \phi(t) / t=\infty, g:[a, b] \times X \rightarrow$ $\mathbb{R}^{1}$ be a continuous function such that

$$
g(t, u) \geqslant \phi(\|u\|) \quad \text { for all }(t, u) \in[a, b] \times X
$$

and let $h:[a, b] \times X \rightarrow[0, \infty)$ be a continuous function. Assume that for $\xi=g, h$ the following property holds:

(A4) For each $M, \epsilon>0$ there exists $\delta>0$ such that

$$
\left|\xi\left(t, x_{1}\right)-\xi\left(t, x_{2}\right)\right| \leqslant \epsilon
$$

for each $t \in[a, b]$ and each $x_{1}, x_{2} \in X$ satisfying

$$
\left\|x_{i}\right\| \leqslant M, \quad i=1,2, \quad\left\|x_{1}-x_{2}\right\| \leqslant \delta
$$

Then (A1)-(A3) hold with the function

$$
f(t, x, u)=h(t, x)+g(t, u), \quad(t, x, u) \in[a, b] \times X \times X .
$$

Propsotion 1.2. Let $\phi:[0, \infty) \rightarrow[0, \infty)$ be an increasing function such that $\lim _{t \rightarrow \infty} \phi(t) / t=\infty, g:[a, b] \times X \rightarrow$ $\mathbb{R}^{1}$ be a continuous function such that

$$
g(t, u) \geqslant \phi(\|u\|) \quad \text { for all }(t, u) \in[a, b] \times X
$$

and let $h:[a, b] \times X \rightarrow[0, \infty)$ be a continuous function such that

$$
\inf \{h(t, x):(t, x) \in[a, b] \times X\}>0 .
$$

Assume that (A4) holds with $\xi=g, h$. Then the function $f(t, x, u)=g(t, u) h(t, x),(t, x, u) \in[a, b] \times X \times X$ satisfies (A1)-(A3).

Corollary 1.1. Let $X=\mathbb{R}^{n}, \phi:[0, \infty) \rightarrow[0, \infty)$ be an increasing function such that

$$
\lim _{t \rightarrow \infty} \phi(t) / t=\infty
$$

$g:[a, b] \times X \rightarrow \mathbb{R}^{1}$ be a continuous function such that

$$
g(t, u) \geqslant \phi(\|u\|) \quad \text { for all }(t, u) \in[a, b] \times X,
$$

let $h:[a, b] \times X \rightarrow[0, \infty)$ be a continuous function such that

$$
\inf \{h(t, x):(t, x) \in[a, b] \times X\}>0
$$


and let

$$
f(t, x, u)=g(t, u) h(t, x), \quad(t, x, u) \in[a, b] \times X \times X .
$$

Then

$$
\inf \{I(v): v \in \mathcal{B}\}=\inf \left\{I(v): v \in \mathcal{B}_{L}\right\} .
$$

It should be mentioned that there are many examples of integrands of the form (1.9) for which the Lavrentiev phenomenon occurs. Corollary 1.1 shows that if such integrands satisfy inequalities (1.7) and (1.8), then the Lavrentiev phenomenon does not occur.

Now we present out second main result.

Let $a, b \in \mathbb{R}^{1}, a<b$. Suppose that $f:[a, b] \times X \times X \rightarrow \mathbb{R}^{1}$ is a continuous function which satisfies the following assumptions:

(B1) There is an increasing function $\phi:[0, \infty) \rightarrow[0, \infty)$ such that

$$
\begin{aligned}
& f(t, x, u) \geqslant \phi(\|u\|) \quad \text { for all }(t, x, u) \in[a, b] \times X \times X, \\
& \lim _{t \rightarrow \infty} \phi(t) / t=\infty .
\end{aligned}
$$

(B2) For each $M>0$ there exist positive numbers $\delta, L$ and an integrable nonnegative scalar function $\psi_{M}(t)$, $t \in[a, b]$, such that for each $t \in[a, b]$, each $u \in X$ and each $x_{1}, x_{2} \in X$ satisfying

$$
\left\|x_{1}\right\|,\left\|x_{2}\right\| \leqslant M, \quad\left\|x_{1}-x_{2}\right\| \leqslant \delta
$$

the following inequality holds:

$$
\left|f\left(t, x_{1}, u\right)-f\left(t, x_{2}, u\right)\right| \leqslant\left\|x_{1}-x_{2}\right\| L\left(f\left(t, x_{1}, u\right)+\psi_{M}(t)\right) .
$$

(B3) For each $M>0$ there is $L>0$ such that for each $t \in[a, b]$ and each $x_{1}, x_{2}, u_{1}, u_{2} \in X$ satisfying $\left\|x_{i}\right\|,\left\|u_{i}\right\| \leqslant$ $M, i=1,2$, the following inequality holds:

$$
\left|f\left(t, x_{1}, u_{1}\right)-f\left(t, x_{2}, u_{2}\right)\right| \leqslant L\left(\left\|x_{1}-x_{2}\right\|+\left\|u_{1}-u_{2}\right\|\right) .
$$

Remark 1.2. It is not difficult to see that if (B2) holds with each $\psi_{M}$ bounded, then $f$ satisfies (A1)-(A3).

For each $z_{1}, z_{2} \in X$ denote by $\mathcal{A}\left(z_{1}, z_{2}\right)$ the set of all $x \in W^{1,1}(a, b ; X)$ such that $x(a)=z_{1}, x(b)=z_{2}$.

For each $x \in \mathcal{A}$ set

$$
I(x)=\int_{a}^{b} f\left(t, x(t), x^{\prime}(t)\right) \mathrm{d} t .
$$

The next theorem is our second main result.

Theorem 1.2. Let $M>0$. Then there exists $K>0$ such that for each $z_{1}, z_{2} \in X$ satisfying $\left\|z_{1}\right\|,\left\|z_{2}\right\| \leqslant M$ and each $x(\cdot) \in \mathcal{A}\left(z_{1}, z_{2}\right)$ the following assertion holds:

If $\operatorname{mes}\left\{t \in[a, b]:\left\|x^{\prime}(t)\right\|>K\right\}>0$, then there exists $y \in \mathcal{A}\left(z_{1}, z_{2}\right)$ such that $I(y)<I(x)$ and $\left\|y^{\prime}(t)\right\| \leqslant K$ for almost every $t \in[a, b]$.

Remark 1.3. (B3) implies that $f$ is bounded on any bounded subset of $[a, b] \times X \times X$.

It is not difficult to see that the following proposition holds. 
Propsotion 1.3. Let $\phi:[0, \infty) \rightarrow[0, \infty)$ be an increasing function such that $\lim _{t \rightarrow \infty} \phi(t) / t=\infty, g:[a, b] \times X \rightarrow$ $\mathbb{R}^{1}$ be a continuous function such that

$$
g(t, u) \geqslant \phi(\|u\|) \quad \text { for all }(t, u) \in[a, b] \times X
$$

and let $h:[a, b] \times X \rightarrow[0, \infty)$ be a continuous function such that

$$
\inf \{h(t, x):(t, x) \in[a, b] \times X\}>0 .
$$

Assume that for $\xi=g$, $h$ the following property holds:

For each $M>0$ there is $L>0$ such that for each $t \in[a, b]$ and each $x_{1}, x_{2}, u_{1}, u_{2} \in X$ satisfying $\left\|x_{i}\right\|,\left\|u_{i}\right\| \leqslant$ $M, i=1,2$, the following inequality holds:

$$
\left|\xi\left(t, x_{1}\right)-\xi\left(t, x_{2}\right)\right| \leqslant L\left\|x_{1}-x_{2}\right\| .
$$

Then (B1)-(B3) hold with the function

$$
f(t, x, u)=h(t, x) g(t, u), \quad(t, x, u) \in[a, b] \times X \times X .
$$

For each $z_{1}, z_{2} \in X$ set

$$
U\left(z_{1}, z_{2}\right)=\inf \left\{I(x): x \in \mathcal{A}\left(z_{1}, z_{2}\right)\right\} .
$$

It is easy to see that $U\left(z_{1}, z_{2}\right)$ is finite for each $z_{1}, z_{2} \in X$.

The paper is organized as follows. Section 2 contains auxiliary results for Theorem 1.1 which is proved in Section 3. In Section 4 we discuss some properties of integrands which satisfy assumptions (A1)-(A3). Our second main result (Theorem 1.2) is proved in Section 6. Section 5 contains auxiliary results for Theorem 1.2.

\section{Auxiliary results}

Set

$$
M_{0}=\inf \{I(v): v \in \mathcal{B}\} .
$$

Clearly $M_{0}$ is a finite number.

Lemma 2.1. There exists a number $M_{1}>0$ such that for each $v \in \mathcal{B}$ satisfying $I(v) \leqslant M_{0}+2$ the following inequality holds:

$$
\|v(t)\| \leqslant M_{1} \quad \text { for all } t \in[a, b]
$$

Proof. Relation (1.2) implies that there is $c_{0} \geqslant 1$ such that

$$
\phi(t) \geqslant t \quad \text { for all } t \geqslant c_{0} .
$$

Assume that $v \in \mathcal{B}$ satisfies

$$
I(v) \leqslant M_{0}+2 .
$$

Let $\tau \in(a, b]$ and

$$
E_{1}=\left\{t \in[a, \tau]:\left\|v^{\prime}(t)\right\| \geqslant c_{0}\right\}, \quad E_{2}=[a, \tau] \backslash E_{1} .
$$

By (2.5) and the definition of $\mathcal{B}$ 


$$
\begin{aligned}
\|v(\tau)\| & =\left\|v(a)+\int_{a}^{\tau} v^{\prime}(t) \mathrm{d} t\right\| \leqslant\|v(a)\|+\int_{a}^{\tau}\left\|v^{\prime}(t)\right\| \mathrm{d} t \\
& \leqslant\left\|z_{1}\right\|+\int_{E_{1}}\left\|v^{\prime}(t)\right\| \mathrm{d} t+\int_{E_{2}}\left\|v^{\prime}(t)\right\| \mathrm{d} t \\
& \leqslant\left\|z_{1}\right\|+c_{0} \operatorname{mes}\left(E_{2}\right)+\int_{E_{1}}\left\|v^{\prime}(t)\right\| \mathrm{d} t \\
& \leqslant\left\|z_{1}\right\|+c_{0}(b-a)+\int_{E_{1}}\left\|v^{\prime}(t)\right\| \mathrm{d} t .
\end{aligned}
$$

We estimate $\int_{E_{1}}\left\|v^{\prime}(t)\right\| \mathrm{d} t$. It follows from (2.5), (2.3) and (1.1) that for all $t \in E_{1}$

$$
\left\|v^{\prime}(t)\right\| \leqslant \phi\left(\left\|v^{\prime}(t)\right\|\right) \leqslant f\left(t, v(t), v^{\prime}(t)\right)
$$

Together with (1.1), (1.6), (2.5) and (2.4) this inequality implies that

$$
\int_{E_{1}}\left\|v^{\prime}(t)\right\| \mathrm{d} t \leqslant \int_{E_{1}} f\left(t, v(t), v^{\prime}(t)\right) \mathrm{d} t \leqslant I(v) \leqslant M_{0}+2 .
$$

Combined with (2.6) this inequality implies that

$$
\|v(\tau)\| \leqslant\left\|z_{1}\right\|+c_{0}(b-a)+M_{0}+2 .
$$

Thus the inequality (2.2) holds with

$$
M_{1}=\left\|z_{1}\right\|+c_{0}(b-a)+M_{0}+2 .
$$

Lemma 2.1 is proved.

Lemma 2.2. Let $\epsilon, M>0$. Then there exist $\Gamma, \delta>0$ such that

$$
\left|f\left(t, x_{1}, u\right)-f\left(t, x_{2}, u\right)\right| \leqslant \epsilon \min \left\{f\left(t, x_{1}, u\right), f\left(t, x_{2}, u\right)\right\}
$$

for each $t \in[a, b]$, each $u \in X$ satisfying $\|u\| \geqslant \Gamma$ and each $x_{1}, x_{2} \in X$ satisfying

$$
\left\|x_{1}\right\|,\left\|x_{2}\right\| \leqslant M, \quad\left\|x_{1}-x_{2}\right\| \leqslant \delta .
$$

Proof. Choose a number $\epsilon_{0} \in(0,1)$ such that

$$
\epsilon_{0}\left(1-\epsilon_{0}\right)^{-1}<\epsilon
$$

By (A2) there exist $\Gamma, \delta>0$ such that

$$
\left|f\left(t, x_{1}, u\right)-f\left(t, x_{2}, u\right)\right| \leqslant \epsilon_{0} \max \left\{f\left(t, x_{1}, u\right), f\left(t, x_{2}, u\right)\right\}
$$

for each $t \in[a, b]$, each $u \in X$ satisfying $\|u\| \geqslant \Gamma$ and each $x_{1}, x_{2} \in X$ satisfying (2.8).

Assume that $t \in[a, b], u \in X$ satisfy $\|u\| \geqslant \Gamma$ and $x_{1}, x_{2} \in X$ satisfy (2.8). Then (2.10) is true. We show that (2.7) holds.

We may assume without loss of generality that

$$
f\left(t, x_{2}, u\right) \geqslant f\left(t, x_{1}, u\right) \text {. }
$$

In view of (2.10) and (2.11)

$$
f\left(t, x_{2}, u\right)-f\left(t, x_{1}, u\right) \leqslant \epsilon_{0} f\left(t, x_{2}, u\right)
$$


and

$$
f\left(t, x_{1}, u\right) \leqslant f\left(t, x_{2}, u\right) \leqslant\left(1-\epsilon_{0}\right)^{-1} f\left(t, x_{1}, u\right) .
$$

Combined with (2.10), (2.11) and (2.9) the inequality (2.12) implies that

$$
\begin{aligned}
\left|f\left(t, x_{1}, u\right)-f\left(t, x_{2}, u\right)\right| & \leqslant \epsilon_{0} f\left(t, x_{2}, u\right) \leqslant \epsilon_{0}\left(1-\epsilon_{0}\right)^{-1} f\left(t, x_{1}, u\right) \\
& \leqslant \epsilon f\left(t, x_{1}, u\right)=\epsilon \min \left\{f\left(t, x_{1}, u\right), f\left(t, x_{2}, u\right)\right\} .
\end{aligned}
$$

This completes the proof of Lemma 2.2.

\section{Proof of Theorem 1.1}

Set

$$
M_{0}=\inf \{I(v): v \in \mathcal{B}\} .
$$

Clearly $M_{0}$ is a finite number. Let $\epsilon \in(0,1)$. In order to prove the theorem it is sufficient to show that for each $v \in \mathcal{B}$ satisfying $I(v) \leqslant M_{0}+1$ there is $u \in \mathcal{B}_{L}$ such that $I(u) \leqslant I(v)+\epsilon$.

By Lemma 2.1 there is $M_{1}>0$ such that

$$
\|v(t)\| \leqslant M_{1}, \quad t \in[a, b],
$$

for all $v \in \mathcal{B}$ satisfying $I(v) \leqslant M_{0}+2$.

Choose a positive number $\epsilon_{0}$ such that

$$
8 \epsilon_{0}\left(M_{0}+4\right)<\epsilon
$$

and a positive number $\gamma_{0}$ such that

$$
\gamma_{0}<1 \text { and } 8 \gamma_{0}\left(M_{0}+2\right)<b-a .
$$

Relation (1.2) implies that there is $N>1$ such that

$$
\phi(t) / t \geqslant \gamma_{0}^{-1} \text { for all } t \geqslant N .
$$

In view of Lemma 2.2 there are

$$
\delta_{0} \in(0,1), \quad N_{0}>N
$$

such that for each $t \in[a, b]$, each $y \in X$ satisfying $\|y\| \geqslant N_{0}$ and each $x_{1}, x_{2} \in X$ satisfying

$$
\left\|x_{1}\right\|,\left\|x_{2}\right\| \leqslant M_{1}+2, \quad\left\|x_{1}-x_{2}\right\| \leqslant \delta_{0}
$$

the following inequality holds:

$$
\left|f\left(t, x_{1}, y\right)-f\left(t, x_{2}, y\right)\right| \leqslant \epsilon_{0} \min \left\{f\left(t, x_{1}, y\right), f\left(t, x_{2}, y\right)\right\} .
$$

By (A3) there exists

$$
\delta_{1} \in\left(0, \delta_{0}\right)
$$

such that

$$
\left|f\left(t, x_{1}, y_{1}\right)-f\left(t, x_{2}, y_{2}\right)\right| \leqslant(8(b-a+1))^{-1} \epsilon
$$

for each $t \in[a, b]$ and each $x_{1}, x_{2}, y_{1}, y_{2} \in X$ satisfying

$$
\left\|x_{1}\right\|,\left\|x_{2}\right\| \leqslant M_{1}+2, \quad\left\|y_{1}\right\|,\left\|y_{2}\right\| \leqslant N_{0}+1, \quad\left\|x_{1}-x_{2}\right\|,\left\|y_{1}-y_{2}\right\| \leqslant \delta_{1} .
$$


It follows from (A3) that we can choose

$$
M_{2}>\sup \left\{f(t, y, 0): t \in[a, b], y \in X \text { and }\|y\| \leqslant M_{1}+1\right\} .
$$

Choose a positive number $\gamma_{1}$ such that

$$
8 \gamma_{1}\left(M_{0}+M_{1}+4\right)<\delta_{1} \min \{1, b-a\} .
$$

By (1.2) there is a number $N_{1}$ such that

$$
N_{1}>N_{0}+M_{2}+4 \text { and } \phi(t) / t \geqslant \gamma_{1}^{-1} \text { for all } t \geqslant N_{1} \text {. }
$$

Assume that

$$
v \in \mathcal{B} \quad \text { and } \quad I(v) \leqslant M_{0}+2 .
$$

It follows from (3.15) and the choice of $M_{1}$ that the inequality (3.2) holds. Set

$$
\begin{aligned}
& E_{1}=\left\{t \in[a, b]:\left\|v^{\prime}(t)\right\| \geqslant N_{1}\right\}, \\
& E_{2}=\left\{t \in[a, b]:\left\|v^{\prime}(t)\right\| \leqslant N_{0}\right\}, \quad E_{3}=[a, b] \backslash\left(E_{1} \cup E_{2}\right) .
\end{aligned}
$$

(3.16), (3.14), (1.1), (1.6) and (3.15) imply that

$$
\begin{aligned}
\left\|\int_{E_{1}} v^{\prime}(t) \mathrm{d} t\right\| & \leqslant \int_{E_{1}}\left\|v^{\prime}(t)\right\| \mathrm{d} t \leqslant \int_{E_{1}} \gamma_{1} \phi\left(\left\|v^{\prime}(t)\right\|\right) \mathrm{d} t \\
& \leqslant \gamma_{1} \int_{E_{1}} f\left(t, v(t), v^{\prime}(t)\right) \mathrm{d} t \leqslant \gamma_{1} I(v) \leqslant \gamma_{1}\left(M_{0}+2\right) .
\end{aligned}
$$

Set

$$
h_{0}=\int_{E_{1}} v^{\prime}(t) \mathrm{d} t .
$$

By (3.17) and (3.18)

$$
\left\|h_{0}\right\| \leqslant \gamma_{1}\left(M_{0}+2\right) \text {. }
$$

Now we estimate mes $\left(E_{2}\right)$. It follows from (3.16), the choice of $N$ (see (3.5)), (3.6), (1.1), (1.6) and (3.15) that

$$
\begin{aligned}
\operatorname{mes}\left(E_{1} \cup E_{3}\right) & \leqslant N_{0}^{-1} \int_{E_{1} \cup E_{3}}\left\|v^{\prime}(t)\right\| \mathrm{d} t \leqslant \gamma_{0} N_{0}^{-1} \int_{E_{1} \cup E_{3}} \phi\left(\left\|v^{\prime}(t)\right\|\right) \mathrm{d} t \\
& \leqslant \gamma_{0} N_{0}^{-1} \int_{E_{1} \cup E_{3}} f\left(t, v(t), v^{\prime}(t)\right) \mathrm{d} t \leqslant \gamma_{0} N_{0}^{-1} I(v) \leqslant \gamma_{0} I(v) \leqslant \gamma_{0}\left(M_{0}+2\right) .
\end{aligned}
$$

Combined with (3.16) and (3.4) this inequality implies that

$$
\operatorname{mes}\left(E_{2}\right) \geqslant b-a-\gamma_{0}\left(M_{0}+2\right) \geqslant(3 / 4)(b-a) .
$$

Define a measurable function $\xi:[a, b] \rightarrow X$ by

$$
\begin{aligned}
& \xi(t)=0, \quad t \in E_{1}, \quad \xi(t)=v^{\prime}(t), \quad t \in E_{3}, \\
& \xi(t)=v^{\prime}(t)+\left(\operatorname{mes}\left(E_{2}\right)\right)^{-1} h_{0}, \quad t \in E_{2} .
\end{aligned}
$$

Clearly the function $\xi$ is Bochner integrable. It follows from (3.16), (3.21), (3.18) and (3.15) that 


$$
\begin{aligned}
& \int_{a}^{b} \xi(t) \mathrm{d} t=\int_{E_{1}} \xi(t) \mathrm{d} t+\int_{E_{2}} \xi(t) \mathrm{d} t+\int_{E_{3}} \xi(t) \mathrm{d} t=\int_{E_{2}} \xi(t) \mathrm{d} t+\int_{E_{3}} \xi(t) \mathrm{d} t \\
& =\int_{E_{2}} v^{\prime}(t) \mathrm{d} t+h_{0}+\int_{E_{3}} v^{\prime}(t) \mathrm{d} t, \\
& \sum_{i=1}^{3} \int_{E_{i}} v^{\prime}(t) \mathrm{d} t=\int_{a}^{b} v^{\prime}(t) \mathrm{d} t=z_{2}-z_{1}
\end{aligned}
$$

Define a function $u:[a, b] \rightarrow X$ by

$$
u(\tau)=\int_{0}^{\tau} \xi(t) \mathrm{d} t+z_{1}, \quad \tau \in[a, b] .
$$

In view of (3.23), (3.22), (3.21) and (3.16) $u \in \mathcal{B}_{L}$.

Now we show that

$$
\|u(t)-v(t)\| \leqslant \delta_{1} \quad \text { for all } t \in[a, b] .
$$

Let $s \in(a, b]$. By (3.15), (3.23), (3.16), (3.21), (3.17) and (3.19)

$$
\begin{aligned}
\|v(s)-u(s)\| & =\left\|\int_{a}^{s}\left[v^{\prime}(t)-\xi(t)\right] \mathrm{d} t\right\| \\
& \leqslant\left\|\int_{[a, s] \cap E_{1}}\left[v^{\prime}(t)-\xi(t)\right] \mathrm{d} t\right\|+\left\|\int_{[a, s] \cap E_{2}}\left[v^{\prime}(t)-\xi(t)\right] \mathrm{d} t\right\|+\left\|\int_{[a, s] \cap E_{3}}\left[v^{\prime}(t)-\xi(t)\right] \mathrm{d} t\right\| \\
& \leqslant \int_{E_{1}}\left\|v^{\prime}(t)\right\| \mathrm{d} t+\left\|h_{0}\right\| \leqslant 2 \gamma_{1}\left(M_{0}+2\right) .
\end{aligned}
$$

Thus for each $s \in[a, b]$

$$
\|v(s)-u(s)\| \leqslant 2 \gamma_{1}\left(M_{0}+2\right) .
$$

Combined with (3.13) this inequality implies (3.24). It follows from (3.24), (3.2), (3.9) and (3.6) that

$$
\|u(t)\| \leqslant M_{1}+1 \quad \text { for all } t \in[a, b] .
$$

We estimate $I(u)-I(v)$. In view of (1.6) and (3.16)

$$
I(u)-I(v)=\sum_{i=1}^{3} \int_{E_{i}}\left[f\left(t, u(t), u^{\prime}(t)\right)-f\left(t, v(t), v^{\prime}(t)\right)\right] \mathrm{d} t .
$$

By (3.23), (3.21), (3.26) and (3.12) for almost every $t \in E_{1}$

$$
f\left(t, u(t), u^{\prime}(t)\right)=f(t, u(t), \xi(t))=f(t, u(t), 0)<M_{2} .
$$

(1.1), (3.16), (3.14) and (3.13) imply that for almost every $t \in E_{1}$

$$
f\left(t, v(t), v^{\prime}(t)\right) \geqslant \phi\left(\left\|v^{\prime}(t)\right\|\right) \geqslant N_{1}>M_{2}+4 .
$$


Combined with (3.28) this inequality implies that

$$
\int_{E_{1}}\left[f\left(t, u(t), u^{\prime}(t)\right)-f\left(t, v(t), v^{\prime}(t)\right)\right] \mathrm{d} t \leqslant 0 .
$$

Let $t \in E_{2}$ and $v^{\prime}(t), u^{\prime}(t)$ exist. It follows from (3.16) that

$$
\left\|v^{\prime}(t)\right\| \leqslant N_{0} \text {. }
$$

(3.23) and (3.21) imply that

$$
u^{\prime}(t)=\xi(t)=v^{\prime}(t)+\left(\operatorname{mes}\left(E_{2}\right)\right)^{-1} h_{0} .
$$

Together with (3.19), (3.20) and (3.13) this equality implies that

$$
\left\|u^{\prime}(t)-v^{\prime}(t)\right\|=\left(\operatorname{mes}\left(E_{2}\right)\right)^{-1}\left\|h_{0}\right\| \leqslant \gamma_{1}\left(M_{0}+2\right) 2(b-a)^{-1}<\frac{\delta_{1}}{4} .
$$

(3.31), (3.30), (3.9) and (3.6) imply that

$$
\left\|u^{\prime}(t)\right\| \leqslant N_{0}+1 \text {. }
$$

By (3.2), (3.26), (3.30) (3.32), (3.31), (3.24) and the choice of $\delta_{1}$ (see (3.10), (3.11))

$$
\left|f\left(t, v(t), v^{\prime}(t)\right)-f\left(t, u(t), u^{\prime}(t)\right)\right| \leqslant(8(b-a+1))^{-1} \epsilon .
$$

Since this inequality holds for almost every $t \in E_{2}$ we obtain that

$$
\left|\int_{E_{2}}\left[f\left(t, u(t), u^{\prime}(t)\right)-f\left(t, v(t), v^{\prime}(t)\right)\right] \mathrm{d} t\right| \leqslant \frac{\epsilon}{8} .
$$

Let $t \in E_{3}$ and $u^{\prime}(t)$ and $v^{\prime}(t)$ exist. By (3.16) and (3.14)

$$
\left\|v^{\prime}(t)\right\| \geqslant N_{0} \text {. }
$$

In view of (3.23) and (3.21)

$$
\left|f\left(t, v(t), v^{\prime}(t)\right)-f\left(t, u(t), u^{\prime}(t)\right)\right|=\left|f\left(t, v(t), v^{\prime}(t)\right)-f\left(t, u(t), v^{\prime}(t)\right)\right| .
$$

It follows from this equality, (3.2), (3.26), (3.24), (3.9), (3.34) and the choice of $\delta_{0}, N_{0}$ (see (3.6)-(3.8)) that

$$
\left|f\left(t, v(t), v^{\prime}(t)\right)-f\left(t, u(t), u^{\prime}(t)\right)\right| \leqslant \epsilon_{0} f\left(t, v(t), v^{\prime}(t)\right) .
$$

By this inequality which holds for almost every $t \in E_{3}$, (3.15) and (3.3)

$$
\left|\int_{E_{3}}\left[f\left(t, u(t), u^{\prime}(t)\right)-f\left(t, v(t), v^{\prime}(t)\right)\right] \mathrm{d} t\right| \leqslant \int_{E_{3}} \epsilon_{0} f\left(t, v(t), v^{\prime}(t)\right) \mathrm{d} t \leqslant \epsilon_{0} I(v) \leqslant \epsilon_{0}\left(M_{0}+2\right)<\frac{\epsilon}{8} .
$$

Combined with (3.29), (3.33) and (3.27) this inequality implies that $I(u)-I(v) \leqslant \epsilon / 2$. This completes the proof of Theorem 1.1.

\section{Properties of integrands which satisfy (A1)-(A3)}

Let $(X,\|\cdot\|)$ be a Banach space and let $a, b \in \mathbb{R}^{1}$ be such that $a<b$. 
Propsotion 4.1. Assume that $g:[a, b] \times X \times X \rightarrow \mathbb{R}^{1}$ is a continuous function, (A1)-(A3) hold with $f=g$ and $h:[a, b] \times X \times X \rightarrow[0, \infty)$ is a continuous function such that (A3) holds with $f=h$ and for each bounded subset $D \subset[a, b] \times X$

$$
\lim _{\|u\| \rightarrow \infty}[h(t, x, u) / g(t, x, u)]=0 \quad \text { uniformly for }(t, x) \in D .
$$

Then (A1)-(A3) hold with $f=g+h$.

Proof. Clearly (A1) and (A3) hold with $f=g+h$. We show that (A2) holds with $f=g+h$.

Let $M, \epsilon>0$. By Lemma 2.2 there exist $\Gamma_{0}, \delta>0$ such that for each $t \in[a, b]$ and each $x_{1}, x_{2}, u \in X$ satisfying

$$
\|u\| \geqslant \Gamma_{0}, \quad\left\|x_{1}\right\|,\left\|x_{2}\right\| \leqslant M, \quad\left\|x_{1}-x_{2}\right\| \leqslant \delta
$$

the following inequality holds:

$$
\left|g\left(t, x_{1}, u\right)-g\left(t, x_{2}, u\right)\right| \leqslant(\epsilon / 8) \min \left\{g\left(t, x_{1}, u\right), g\left(t, x_{2}, u\right)\right\} .
$$

In view of (4.1) there is

$$
\Gamma>\Gamma_{0}
$$

such that for each $t \in[a, b]$ and each $x, u \in X$ satisfying

$$
\|x\| \leqslant M, \quad\|u\| \geqslant \Gamma
$$

the following inequality holds:

$$
h(t, x, u) \leqslant(\epsilon / 8) g(t, x, u) .
$$

Assume now that $t \in[a, b]$ and $x_{1}, x_{2}, u \in X$ satisfy

$$
\left\|x_{1}\right\|,\left\|x_{2}\right\| \leqslant M, \quad\left\|x_{1}-x_{2}\right\| \leqslant \delta, \quad\|u\| \geqslant \Gamma .
$$

By (4.7), (4.4) and the choice of $\Gamma_{0}, \delta$ the inequality (4.3) holds. It follows from (4.7) and the choice of $\Gamma$ (see (4.4)-(4.6)) that

$$
h\left(t, x_{i}, u\right) \leqslant(\epsilon / 8) g\left(t, x_{i}, u\right), \quad i=1,2 .
$$

(4.3) implies that

$$
\begin{aligned}
\left|(g+h)\left(t, x_{2}, u\right)-(g+h)\left(t, x_{1}, u\right)\right| & \leqslant\left|g\left(t, x_{1}, u\right)-g\left(t, x_{2}, u\right)\right|+\left|h\left(t, x_{1}, u\right)-h\left(t, x_{2}, u\right)\right| \\
& \leqslant(\epsilon / 8) \min \left\{g\left(t, x_{1}, u\right), g\left(t, x_{2}, u\right)\right\}+\left|h\left(t, x_{1}, u\right)-h\left(t, x_{2}, u\right)\right| .
\end{aligned}
$$

We may assume without loss of generality that

$$
h\left(t, x_{2}, u\right) \geqslant h\left(t, x_{1}, u\right) .
$$

In view of (4.10), (4.9) and (4.8)

$$
\left|(g+h)\left(t, x_{2}, u\right)-(g+h)\left(t, x_{1}, u\right)\right| \leqslant(\epsilon / 8) g\left(t, x_{2}, u\right)+h\left(t, x_{2}, u\right) \leqslant(\epsilon / 8) g\left(t, x_{2}, u\right)+(\epsilon / 8) g\left(t, x_{2}, u\right) .
$$

Hence (A2) holds with $f=g+h$. This completes the proof of Proposition 4.1.

It is easy to see that if $g:[a, b] \times X \times X \rightarrow \mathbb{R}^{1}$ is a continuous function, (A1)-(A3) hold with $f=g$ and $\lambda>0$, then (A1)-(A3) hold with $f=\lambda g$.

Propsotion 4.2. Let $g_{1}, g_{2}:[a, b] \times X \times X \rightarrow \mathbb{R}^{1}$ be a continuous function such that (A1)-(A3) hold with $f=g_{1}, g_{2}$. Then (A1)-(A3) hold with $f=g_{1}+g_{2}$. 
Proof. Clearly (A1) and (A3) hold with $f=g_{1}+g_{2}$. Let us show that (A2) holds with $f=g_{1}+g_{2}$.

Let $\epsilon, M>0$. By Lemma 2.2 there are $\Gamma, \delta>0$ such that for each $t \in[a, b]$, each $u \in X$ satisfying $\|u\| \geqslant \Gamma$ and each $x_{1}, x_{2} \in X$ satisfying

$$
\left\|x_{1}\right\|,\left\|x_{2}\right\| \leqslant M, \quad\left\|x_{1}-x_{2}\right\| \leqslant \delta
$$

we have

$$
\left|g_{i}\left(t, x_{1}, u\right)-g_{i}\left(t, x_{2}, u\right)\right| \leqslant \epsilon \min \left\{g_{i}\left(t, x_{1}, u\right), g_{2}\left(t, x_{2}, u\right)\right\}, \quad i=1,2 .
$$

Let $t \in[a, b], u \in X,\|u\| \geqslant \Gamma, x_{1}, x_{2} \in X$ satisfy (4.11). Then (4.12) holds and

$$
\left|\left(g_{1}+g_{2}\right)\left(t, x_{1}, u\right)-\left(g_{1}+g_{2}\right)\left(t, x_{2}, u\right)\right| \leqslant \epsilon g_{1}\left(t, x_{1}, u\right)+\epsilon g_{2}\left(t, x_{1}, u\right) .
$$

Hence (A2) holds with $f=g_{1}+g_{2}$. This completes the proof of Proposition 4.2.

\section{Auxiliary results for Theorem 1.2}

For each $z_{1}, z_{2} \in X$ set

$$
U\left(z_{1}, z_{2}\right)=\inf \left\{I(x): x \in \mathcal{A}\left(z_{1}, z_{2}\right)\right\} .
$$

It is easy to see that $U\left(z_{1}, z_{2}\right)$ is finite for each $z_{1}, z_{2} \in X$.

Lemma 5.1. Let $M>0$. Then there is $M_{1}>0$ such that

$$
U\left(z_{1}, z_{2}\right) \leqslant M_{1} \text { for each } z_{1}, z_{2} \in X \text { satisfying }\left\|z_{1}\right\|,\left\|z_{2}\right\| \leqslant M .
$$

Proof. Set

$$
M_{1}=\sup \left\{f(s, z, u): s \in[a, b], z, u \in X \text { and }\|z\|,\|u\| \leqslant 2 M\left(1+(b-a)^{-1}\right)\right\}(b-a) .
$$

By Remark $1.3 M_{1}$ is finite. Assume that $z_{1}, z_{2} \in X$ and

$$
\left\|z_{1}\right\|,\left\|z_{2}\right\| \leqslant M \text {. }
$$

Define $x \in \mathcal{A}\left(z_{1}, z_{2}\right)$ by

$$
x(t)=z_{1}+(t-a)(b-a)^{-1}\left(z_{2}-z_{1}\right), \quad t \in[a, b] .
$$

Clearly

$$
\|x(t)\| \leqslant M \quad \text { for all } t \in[a, b] .
$$

It is easy to see that for all $t \in[a, b]$

$$
\left\|x^{\prime}(t)\right\| \leqslant(b-a)^{-1}\left\|z_{2}-z_{1}\right\| \leqslant 2 M(b-a)^{-1} .
$$

(5.4), (5.5) and (5.1) imply that for all $t \in[a, b]$

$$
f\left(t, x(t), x^{\prime}(t)\right) \leqslant M_{1} /(b-a) .
$$

This inequality implies that

$$
U\left(z_{1}, z_{2}\right) \leqslant I(x) \leqslant M_{1} .
$$

Lemma 5.1 is proved. 
Lemma 5.2. Let $M>0$. Then there is $M_{0}>0$ such that for each $z_{1}, z_{2} \in X$ satisfying $\left\|z_{1}\right\|,\left\|z_{2}\right\| \leqslant M$ and each $x \in \mathcal{A}\left(z_{1}, z_{2}\right)$ satisfying $I(x) \leqslant U\left(z_{1}, z_{2}\right)+1$ the inequality $\|x(t)\| \leqslant M_{0}$ holds for all $t \in[a, b]$.

Proof. By Lemma 5.1 there is $M_{1}>0$ such that

$$
U\left(z_{1}, z_{2}\right) \leqslant M_{1} \text { for each } z_{1}, z_{2} \in X \text { satisfying }\left\|z_{1}\right\|,\left\|z_{2}\right\| \leqslant M .
$$

(1.12) implies that there is $c_{0} \geqslant 1$ such that

$$
\phi(t) \geqslant t \quad \text { for all } t \geqslant c_{0} .
$$

Set

$$
M_{0}=M+M_{1}+1+c_{0}(b-a) .
$$

Assume that $z_{1}, z_{2} \in X, x \in \mathcal{A}\left(z_{1}, z_{2}\right)$,

$$
\left\|z_{1}\right\|,\left\|z_{2}\right\| \leqslant M, \quad I(x) \leqslant U\left(z_{1}, z_{2}\right)+1 .
$$

In view of (5.9) and the choice of $M_{1}$ (see (5.6))

$$
U\left(z_{1}, z_{2}\right) \leqslant M_{1} \text { and } I(x) \leqslant M_{1}+1 .
$$

Let $\tau \in(a, b]$ and set

$$
E_{1}=\left\{t \in[a, \tau]:\left\|x^{\prime}(t)\right\| \geqslant c_{0}\right\}, \quad E_{2}=[a, \tau] \backslash E_{1} .
$$

By (5.11) and (5.9)

$$
\begin{aligned}
\|x(\tau)\| & =\left\|x(a)+\int_{a}^{\tau} x^{\prime}(t) \mathrm{d} t\right\| \leqslant\|x(a)\|+\int_{a}^{\tau}\left\|x^{\prime}(t)\right\| \mathrm{d} t \leqslant\left\|z_{1}\right\|+\int_{E_{1}}\left\|x^{\prime}(t)\right\| \mathrm{d} t+\int_{E_{2}}\left\|x^{\prime}(t)\right\| \mathrm{d} t \\
& \leqslant M+c_{0} \operatorname{mes}\left(E_{2}\right)+\int_{E_{1}}\left\|x^{\prime}(t)\right\| \mathrm{d} t \leqslant(b-a) c_{0}+M+\int_{E_{1}}\left\|x^{\prime}(t)\right\| \mathrm{d} t .
\end{aligned}
$$

It follows from (5.11), (5.7), (1.11) and (5.10) that

$$
\int_{E_{1}}\left\|x^{\prime}(t)\right\| \mathrm{d} t \leqslant \int_{E_{1}} \phi\left(\left\|x^{\prime}(t)\right\|\right) \mathrm{d} t \leqslant \int_{a}^{b} \phi\left(\left\|x^{\prime}(t)\right\|\right) \mathrm{d} t \leqslant I(x) \leqslant M_{1}+1 .
$$

Combined with (5.12) and (5.8) this implies that

$$
\|x(\tau)\| \leqslant(b-a) c_{0}+M+M_{1}+1=M_{0} .
$$

This completes the proof of Lemma 5.2.

\section{Proof of Theorem 1.2}

Let $M>0$. By Lemma 5.1 there is $M_{1}>0$ such that

$$
U\left(z_{1}, z_{2}\right) \leqslant M_{1} \text { for each } z_{1}, z_{2} \in X \text { satisfying }\left\|z_{1}\right\|,\left\|z_{2}\right\| \leqslant M .
$$

In view of Lemma 5.2 there is $M_{0}>0$ such that for each $z_{1}, z_{2} \in X$ and each $x \in \mathcal{A}\left(z_{1}, z_{2}\right)$ satisfying

$$
\left\|z_{1}\right\|,\left\|z_{2}\right\| \leqslant M, \quad I(x) \leqslant U\left(z_{1}, z_{2}\right)+1
$$


the following inequality holds:

$$
\|x(t)\| \leqslant M_{0}, \quad t \in[a, b] .
$$

By (B2) there are $\delta_{0}, L_{0}>0$ and an integrable scalar function $\psi_{0}(t) \geqslant 0, t \in[a, b]$ such that for each $t \in[a, b]$, each $u \in X$ and each $x_{1}, x_{2} \in X$ satisfying

$$
\left\|x_{1}\right\|,\left\|x_{2}\right\| \leqslant M_{0}+4, \quad\left\|x_{1}-x_{2}\right\| \leqslant \delta_{0}
$$

the following inequality holds:

$$
\left|f\left(t, x_{1}, u\right)-f\left(t, x_{2}, u\right)\right| \leqslant\left\|x_{1}-x_{2}\right\| L_{0}\left(f\left(t, x_{1}, u\right)+\psi_{0}(t)\right) .
$$

Choose a positive number $\gamma_{0}$ such that

$$
\gamma_{0}<1 \text { and } 8 \gamma_{0}\left(M_{1}+1\right)<b-a \text {. }
$$

(1.12) implies that there is $K_{0}>1$ such that

$$
\phi(t) / t \geqslant \gamma_{0}^{-1} \text { for all } t \geqslant K_{0} .
$$

Set

$$
\Delta_{0}=\sup \left\{f(t, z, 0): t \in[a, b], z \in X,\|z\| \leqslant M_{0}+2\right\} .
$$

Remark 1.3 implies that $\Delta_{0}$ is finite.

It follows from (B3) that there is $L_{1}>1$ such that for each $t \in[a, b]$ and each $x_{1}, x_{2}, u_{1}, u_{2} \in X$ satisfying

$$
\left\|x_{1}\right\|,\left\|x_{2}\right\|,\left\|u_{1}\right\|,\left\|u_{2}\right\| \leqslant K_{0}+M_{0}+2
$$

the following inequality holds:

$$
\left|f\left(t, x_{1}, u_{1}\right)-f\left(t, x_{2}, u_{2}\right)\right| \leqslant L_{1}\left(\left\|x_{1}-x_{2}\right\|+\left\|u_{1}-u_{2}\right\|\right) .
$$

Choose a number $\gamma_{1} \in(0,1)$ such that

$$
\begin{aligned}
& 2 \gamma_{1}\left(M_{1}+2\right)<(\min \{1, b-a\}) \min \left\{1, \delta_{0} / 4\right\}, \\
& \gamma_{1}<\left(16 L_{1}\left(b-a+1+\int_{a}^{b} \psi_{0}(t) \mathrm{d} t\right)+64+16 L_{0}\left(b-a+1+M_{1}+\int_{a}^{b} \psi_{0}(t) \mathrm{d} t\right)\right)^{-1} .
\end{aligned}
$$

By (1.12) there is a number $K>0$ such that

$$
\begin{aligned}
& K>8 \Delta_{0}+K_{0}+2, \\
& \phi(t) / t \geqslant \gamma_{1}^{-1} \quad \text { for all } t \geqslant K .
\end{aligned}
$$

Assume that

$$
\begin{aligned}
& z_{1}, z_{2} \in X, \quad\left\|z_{1}\right\|,\left\|z_{2}\right\| \leqslant M, \\
& x \in \mathcal{A}\left(z_{1}, z_{2}\right), \\
& \operatorname{mes}\left\{t \in[a, b]:\left\|x^{\prime}(t)\right\|>K\right\}>0 .
\end{aligned}
$$

We show that there is $u \in \mathcal{A}\left(z_{1}, z_{2}\right)$ such that $I(u)<I(x)$ and $\left\|u^{\prime}(t)\right\| \leqslant K$ for almost every $t \in[a, b]$.

We may assume without loss of generality that

$$
I(x) \leqslant U\left(z_{1}, z_{2}\right)+1 .
$$

(6.18), (6.1) and (6.15) imply that

$$
I(x) \leqslant M_{1}+1 .
$$


In view of (6.15), (6.16), (6.18) and the choice of $M_{0}$ (see (6.2), (6.3))

$$
\|x(t)\| \leqslant M_{0}, t \in[a, b]
$$

Set

$$
E_{1}=\left\{t \in[a, b]:\left\|x^{\prime}(t)\right\| \geqslant K\right\}, \quad E_{2}=\left\{t \in[a, b]:\left\|x^{\prime}(t)\right\| \leqslant K_{0}\right\}, \quad E_{3}=[a, b] \backslash\left(E_{1} \cup E_{2}\right) .
$$

Set

$$
\begin{aligned}
& d=\int_{E_{1}}\left\|x^{\prime}(t)\right\| \mathrm{d} t, \\
& h_{0}=\int_{E_{1}} x^{\prime}(t) \mathrm{d} t .
\end{aligned}
$$

It follows from (6.22), (6.21) and (6.17) that

$$
d>0 .
$$

Clearly

$$
\left\|h_{0}\right\| \leqslant d \text {. }
$$

By (6.22), (6.21), (6.14), (1.11) and (6.19)

$$
\begin{aligned}
d & =\int_{E_{1}}\left\|x^{\prime}(t)\right\| \mathrm{d} t \leqslant \int_{E_{1}} \gamma_{1} \phi\left(\left\|x^{\prime}(t)\right\|\right) \mathrm{d} t \leqslant \gamma_{1} \int_{a}^{b} \phi\left(\left\|x^{\prime}(t)\right\|\right) \mathrm{d} t \\
& \leqslant \gamma_{1} \int_{a}^{b} f\left(t, x(t), x^{\prime}(t)\right) \mathrm{d} t \leqslant \gamma_{1}\left(M_{1}+1\right) .
\end{aligned}
$$

Now we estimate mes $\left(E_{2}\right)$. It follows from (6.21), (6.7), (6.13), (1.11) and (6.19) that

$$
\begin{aligned}
\operatorname{mes}\left(E_{1} \cup E_{3}\right) & \leqslant K_{0}^{-1} \int_{E_{1} \cup E_{3}}\left\|x^{\prime}(t)\right\| \mathrm{d} t \leqslant K_{0}^{-1} \int_{E_{1} \cup E_{3}} \gamma_{0} \phi\left(\left\|x^{\prime}(t)\right\|\right) \mathrm{d} t \leqslant \gamma_{0} K_{0}^{-1} \int_{a}^{b} \phi\left(\left\|x^{\prime}(t)\right\|\right) \mathrm{d} t \\
& \leqslant \gamma_{0} \int_{a}^{b} \phi\left(\left\|x^{\prime}(t)\right\|\right) \mathrm{d} t \leqslant \gamma_{0} \int_{a}^{b} f\left(t, x(t), x^{\prime}(t)\right) \mathrm{d} t \leqslant \gamma_{0}\left(M_{1}+1\right) .
\end{aligned}
$$

Together with (6.21) this inequality implies that

$$
\operatorname{mes}\left(E_{2}\right) \geqslant b-a-\gamma_{0}\left(M_{1}+1\right) \text {. }
$$

(6.28) and (6.6) imply that

$$
\operatorname{mes}\left(E_{2}\right) \geqslant 3(b-a) / 4 \text {. }
$$

Define a measurable function $\xi:[a, b] \rightarrow X$ by

$$
\xi(t)=0, \quad t \in E_{1}, \quad \xi(t)=x^{\prime}(t), \quad t \in E_{3}, \quad \xi(t)=x^{\prime}(t)+\left(\operatorname{mes}\left(E_{2}\right)\right)^{-1} h_{0}, \quad t \in E_{2} .
$$

Clearly $\xi$ is a Bochner integrable function. It follows from (6.30), (6.21), (6.23) and (6.16) that 


$$
\begin{aligned}
& \int_{a}^{b} \xi(t) \mathrm{d} t=\sum_{i=1}^{3} \int_{E_{i}} \xi(t) \mathrm{d} t=\int_{E_{2}} \xi(t) \mathrm{d} t+\int_{E_{3}} \xi(t) \mathrm{d} t \\
& \int_{E_{2}} x^{\prime}(t) \mathrm{d} t+h_{0}+\int_{E_{3}} x^{\prime}(t) \mathrm{d} t=\int_{a}^{b} x^{\prime}(t) \mathrm{d} t=z_{2}-z_{1} .
\end{aligned}
$$

Define a function $u:[a, b] \rightarrow X$ by

$$
u(\tau)=z_{1}+\int_{a}^{\tau} \xi(t) \mathrm{d} t, \quad \tau \in[a, b] .
$$

By (6.31)

$$
u \in \mathcal{A}\left(z_{1}, z_{2}\right) .
$$

In view of (6.32), (6.30), (6.29) and (6.25) for almost every $t \in E_{2}$

$$
\left\|x^{\prime}(t)-u^{\prime}(t)\right\|=\left\|x^{\prime}(t)-\xi(t)\right\|=\left(\operatorname{mes}\left(E_{2}\right)\right)^{-1}\left\|h_{0}\right\| \leqslant 2(b-a)^{-1} d .
$$

Combined with (6.26), (6.21) and (6.11) this relation implies that for almost every $t \in E_{2}$

$$
\left\|u^{\prime}(t)\right\| \leqslant\left\|x^{\prime}(t)\right\|+2(b-a)^{-1} d \leqslant\left\|x^{\prime}(t)\right\|+2(b-a)^{-1} \gamma_{1}\left(M_{1}+1\right) \leqslant K_{0}+1 .
$$

(6.35), (6.13), (6.21), (6.30) and (6.32) imply that

$$
\left\|u^{\prime}(t)\right\| \leqslant K \quad \text { for almost every } t \in[a, b] .
$$

We show that $I(u)<I(x)$.

Let $s \in(a, b]$. It follows from (6.32), (6.16), (6.21), (6.30), (6.25) and (6.22) that

$$
\begin{aligned}
\|x(s)-u(s)\| & =\left\|\int_{a}^{s}\left[x^{\prime}(t)-u^{\prime}(t)\right] \mathrm{d} t\right\|=\left\|\int_{a}^{s}\left[x^{\prime}(t)-\xi(t)\right] \mathrm{d} t\right\| \\
& \leqslant\left\|\int_{[a, s] \cap E_{1}}\left[x^{\prime}(t)-\xi(t)\right] \mathrm{d} t\right\|+\left\|\int_{[a, s] \cap E_{2}}\left[x^{\prime}(t)-\xi(t)\right] \mathrm{d} t\right\|+\left\|\int_{[a, s] \cap E_{3}}\left[x^{\prime}(t)-\xi(t)\right] \mathrm{d} t\right\| \\
& \leqslant \int_{E_{1}}\left\|x^{\prime}(t)\right\| \mathrm{d} t+\left\|h_{0}\right\| \leqslant 2 d .
\end{aligned}
$$

Therefore

$$
\|x(s)-u(s)\| \leqslant 2 d \quad \text { for all } s \in[a, b] .
$$

In view of (6.21)

$$
I(u)-I(x)=\sum_{i=1}^{3} \int_{E_{i}}\left[f\left(t, u(t), u^{\prime}(t)\right)-f\left(t, x(t), x^{\prime}(t)\right) \mathrm{d} t\right] .
$$

By (6.32), (6.30), (6.37), (6.20), (6.26) and (6.11) for almost every $t \in E_{1}$

$$
\begin{aligned}
f\left(t, u(t), u^{\prime}(t)\right) & =f(t, u(t), 0) \leqslant \sup \left\{f(t, z, 0): z \in X,\|z\| \leqslant M_{0}+2 d\right\} \\
& \leqslant \sup \left\{f(t, z, 0): z \in X,\|z\| \leqslant M_{0}+2\right\} .
\end{aligned}
$$


Combined with (6.8) this inequality implies that for almost every $t \in E_{1}$

$$
f\left(t, u(t), u^{\prime}(t)\right) \leqslant \Delta_{0} .
$$

It follows from (1.11), (6.21), (6.14) and (6.13) that for almost every $t \in E_{1}$

$$
f\left(t, x(t), x^{\prime}(t)\right) \geqslant \phi\left(\left\|x^{\prime}(t)\right\|\right) \geqslant\left\|x^{\prime}(t)\right\| \geqslant K>8 \Delta_{0} .
$$

Together with (6.39) this inequality implies that for almost every $t \in E_{1}$

$$
f\left(t, x(t), x^{\prime}(t)\right)-f\left(t, u(t), u^{\prime}(t)\right) \geqslant 3 f\left(t, x(t), x^{\prime}(t)\right) / 4 .
$$

(6.40) implies that

$$
\int_{E_{1}}\left[f\left(t, u(t), u^{\prime}(t)\right)-f\left(t, x(t), x^{\prime}(t)\right)\right] \mathrm{d} t \leqslant-\frac{3}{4} \int_{E_{1}} f\left(t, x(t), x^{\prime}(t)\right) \mathrm{d} t .
$$

By (6.20), (6.37), (6.26) and (6.11) for all $t \in[a, b]$

$$
\|u(t)\| \leqslant\|x(t)\|+2 d \leqslant M_{0}+2 \gamma_{1}\left(M_{1}+1\right) \leqslant M_{0}+1 .
$$

It follows from (6.20), (6.42), (6.35), (6.21) and the choice $L_{1}$ (see (6.9), (6.10)) that for almost every $t \in E_{2}$

$$
\left|f\left(t, x(t), x^{\prime}(t)\right)-f\left(t, u(t), u^{\prime}(t)\right)\right| \leqslant L_{1}\left(\|x(t)-u(t)\|+\left\|x^{\prime}(t)-u^{\prime}(t)\right\|\right) .
$$

Combined with (6.37) and (6.34) this inequality implies that for almost every $t \in E_{2}$

$$
\left|f\left(t, x(t), x^{\prime}(t)\right)-f\left(t, u(t), u^{\prime}(t)\right)\right| \leqslant L_{1}\left(2 d+2(b-a)^{-1} d\right) .
$$

Therefore

$$
\left|\int_{E_{2}}\left[f\left(t, x(t), x^{\prime}(t)\right)-f\left(t, u(t), u^{\prime}(t)\right)\right] \mathrm{d} t\right| \leqslant 2 d L_{1}(1+b-a) .
$$

(6.37), (6.11) and (6.26) imply that for all $t \in[a, b]$

$$
\|x(t)-u(t)\| \leqslant 2 d \leqslant 2 \gamma_{1}\left(M_{1}+1\right)<\delta_{0} / 4
$$

By (6.44), (6.42), (6.20), (6.30), the choice of $\delta_{0}, L_{0}, \psi_{0}$ (see (6.4), (6.5), (6.30)) and (6.32) for all $t \in E_{3}$

$$
\begin{aligned}
\left|f\left(t, x(t), x^{\prime}(t)\right)-f\left(t, u(t), u^{\prime}(t)\right)\right| & =\left|f\left(t, x(t), x^{\prime}(t)\right)-f\left(t, u(t), x^{\prime}(t)\right)\right| \\
& \leqslant\|x(t)-u(t)\| L_{0}\left(f\left(t, x(t), x^{\prime}(t)\right)+\psi_{0}(t)\right) .
\end{aligned}
$$

Together with (6.37) this inequality implies that for almost all $t \in E_{3}$

$$
\left|f\left(t, x(t), x^{\prime}(t)\right)-f\left(t, u(t), u^{\prime}(t)\right)\right| \leqslant 2 d L_{0}\left(f\left(t, x(t), x^{\prime}(t)\right)+\psi_{0}(t)\right) .
$$

Therefore combining with (6.19) this implies that

$$
\begin{aligned}
\left|\int_{E_{3}}\left[f\left(t, x(t), x^{\prime}(t)\right)-f\left(t, u(t), u^{\prime}(t)\right)\right] \mathrm{d} t\right| & \leqslant 2 d L_{0} \int_{E_{3}}\left(f\left(t, x(t), x^{\prime}(t)\right)+\psi_{0}(t)\right) \mathrm{d} t \\
& \leqslant 2 d L_{0}\left(I(x)+\int_{a}^{b} \psi_{0}(t) \mathrm{d} t\right) \\
& \leqslant 2 d L_{0}\left(M_{1}+1+\int_{a}^{b} \psi_{0}(t) \mathrm{d} t\right) .
\end{aligned}
$$


(6.38), (6.41), (6.43) and (6.45) imply that

$$
I(u)-I(x) \leqslant-(3 / 4) \int_{E_{1}} f\left(t, x(t), x^{\prime}(t)\right) \mathrm{d} t+2 d L_{1}(1+b-a)+2 d L_{0}\left(M_{1}+1+\int_{a}^{b} \psi_{0}(t) \mathrm{d} t\right) .
$$

It follows from (1.11), (6.21) and (6.14) that for all $t \in E_{1}$

$$
f\left(t, x(t), x^{\prime}(t)\right) \geqslant \phi\left(\left\|x^{\prime}(t)\right\|\right) \geqslant \gamma_{1}^{-1}\left\|x^{\prime}(t)\right\| .
$$

Combined with (6.22) and (6.12) this inequality implies that

$$
\begin{aligned}
\int_{E_{1}} f\left(t, x(t), x^{\prime}(t)\right) \mathrm{d} t & \geqslant \gamma_{1}^{-1} \int_{E_{1}}\left\|x^{\prime}(t)\right\| \mathrm{d} t=\gamma_{1}^{-1} d \\
& >d\left(16 L_{1}(b-a+1)+64+16 L_{0}\left(b-a+1+M_{1}+\int_{a}^{b} \psi_{0}(t) \mathrm{d} t\right)\right) .
\end{aligned}
$$

Together with (6.46) this implies that $I(u)-I(x)<0$. Theorem 1.2 is proved.

\section{References}

[1] G. Alberti, F. Serra Cassano, Non-occurrence of gap for one-dimensional autonomous functionals, in: Calculus of Variations, Homogenization and Continuum Mechanics (Marseille, 1993), in: Ser. Adv. Math. Appl. Sci., vol. 18, World Sci., River Edge, NJ, 1994, pp. 1-17.

[2] T.S. Angell, A note on the approximation of optimal solutions of the calculus of variations, Rend. Circ. Mat. Palermo 2 (1979) $258-272$.

[3] J.M. Ball, V.J. Mizel, Singular minimizers for regular one-dimensional problems in the calculus of variations, Bull. Amer. Math. Soc. 11 (1984) 143-146.

[4] J.M. Ball, V.J. Mizel, One-dimensional variational problems whose minimizers do not satisfy the Euler-Lagrange equation, Arch. Rational Mech. Anal. 90 (1985) 325-388.

[5] H. Brezis, Opérateurs Maximaux Monotones et Semi-Groupes de Contractions dans les Espaces de Hilbert, North-Holland, Amsterdam, 1973.

[6] L. Cesari, Optimization - Theory and Applications, Springer-Verlag, Berlin, 1983.

[7] F.H. Clarke, R.B. Vinter, Regularity properties of solutions to the basic problem in the calculus of variations, Trans. Amer. Math. Soc. 289 (1985) 73-98.

[8] F.H. Clarke, R.B. Vinter, Regularity of solutions to variational problems with polynomial Lagrangians, Bull. Polish Acad. Sci. 34 (1986) 73-81.

[9] M. Lavrentiev, Sur quelques problemes du calcul des variations, Ann. Math. Pura Appl. 4 (1926) 107-124.

[10] P.D. Loewen, On the Lavrentiev phenomenon, Canad. Math. Bull. 30 (1987) 102-108.

[11] B. Mania, Sopra un esempio di Lavrentieff, Boll. Un. Mat. Ital. 13 (1934) 146-153.

[12] M.A. Sychev, V.J. Mizel, A condition on the value function both necessary and sufficient for full regularity of minimizers of onedimensional variational problems, Trans. Amer. Math. Soc. 350 (1998) 119-133. 